\title{
Nonstandard finite difference method for ODEs for initial-value problems
}

\author{
Tarik Celik ${ }^{1}$ \\ ${ }^{1}$ Department of Mathematics Education, Faculty of Education Bayburt University, Bayburt, Turkey
}

Received: 12 April 2016, Accepted: 17 July 2016

Published online: 8 October 2016.

\begin{abstract}
In this paper, a powerful recent non-standard finite different method by nonlocal approximation is improved. Also, compared standard finite difference method to this non-standard finite different method in terms of stability and accuracy. As a numerical example, Hybrid Selection \& Genetics equation is considered as the candidate from class of first order ODEs with polynomial right-hand sides. Furthermore, results obtained from the non-standard finite different method and MATLAB ODE solvers (ode15s,ode23s) compared in terms of stability, accuracy, and execution time.
\end{abstract}

Keywords: Nonstandard finite difference method, Nonlocal approximation, MATLAB ODE solvers, Initial-value problems

\section{Introduction}

Ordinary differential equations have significant influence in almost all scientific fields such that Biology, Chemistry, Economics, Engineering, and Physics. Therefore, solving ODEs become very important. There are lots of numerical methods to solve ODEs such that Euler method, Runge - Kutta method or many of others. Also, there are ODE solvers in MATLAB such ode 45, ode15s and ode23s etc. However, all these standard methods do not have time step freedom. But, time step freedom is provided by the non-standard finite difference method that is mentioned in the paper. Interest of the paper is to improve the non-standard finite different method that showed in [1]. R. Anguelov and J.M.-S. Lubuma interest is non-linear terms of ODEs. But, linear term is also taken in the account in this paper. To show idea, the initial value problem for an autonomous first order ordinary differential equation with third degree polynomial right-hand sides is considered.

$$
\frac{d y}{d t}=f(y), y\left(t_{0}\right)=y_{0}
$$

where the function $y \equiv y(t):\left[t_{0}, T\right) \rightarrow R$ is known, $y_{0} \in R$ and the function $f: R \rightarrow R$ given. Here, T could be $\infty$ but $t_{0}$ is finite.

As in shown in [1], numerical approximation of (1) is replace the continuous interval $\left[t_{0}, T\right)$ by the mesh of discrete points $\left\{t_{k}-t_{0}+k h \mid k \geq 0\right\}$ where $h>0$ is the step size. $y_{k} \equiv y\left(t_{k}\right)$ is the solution at $t_{k}$. After applying finite difference method to $(1)$, the sequence $\left(y_{k}\right)$ will be solution of a finite difference equation of the form

$$
y_{k+1}=F\left(h ; y_{k}\right) \text {. }
$$

\footnotetext{
*Corresponding author e-mail: tcelik@bayburt.edu.tr
} 
Definition 1.The equation (2) is called a non-standard finite difference method (NSFDM) if at least one of the following conditions is satisfied:

$$
\frac{d y}{d t}=\frac{\left(y_{k+1}-y_{k}\right)}{\varphi(h)}
$$

where $\varphi(h)$ is non negative function such that $\varphi(h)=h+O\left(h^{2}\right)$ as $h \rightarrow 0$.

Nonlinear terms in $f(y)$ are approximated in a nonlocal way, i.e. by a function of several points of the mesh [1].

Definition 2.Assume that the solution of (1) satisfies some properties. If the numerical scheme (2) satisfies same properties then (2) is called stable with respect to the properties such that monotonicity, boundedness, equilibrium points, and positivity.

In this paper, monotonicity and equilibrium points will be focused on. By [2-4] and the above definition, the NSFDM is more powerful than the standard ones.

\section{Numerical method}

Assume that the function $F(h, y)$ in (2) has continuous derivatives with respect to both variables for $h>0, y \in R$ satisfies

$$
F(0 ; y)=y, \frac{\partial F}{\partial h}(0 ; y)=f(y)
$$

Let us note that consistency implies that (4) is satisfied when $y$ is the solution of (1) (see $[5,6])$.

Theorem 1.The difference equation (2) is stable with respect to monotone dependence on initial value if

$$
\frac{\partial F}{\partial h}(h ; y) \geq 0
$$

where $y \in R, h>0$

Theorem 2.Assume that the difference equation (2) is stable with respect to monotone dependence on initial value. Assume also that for every $h>0$ the equations $F(h ; y)=y$ and $f(y)=0$ in y have the same roots considered with their multiplicity. Then the difference equation (2) is stable with respect to monotonicity of solutions.

Theorem 3.Under the assumptions of theorem 2, the difference equation (2) is elementary stable. Theorem 1, 2 and 3 stated and proved in [1].

\section{Scheme 1.}

For the scheme 1, just nonlinear terms will be formalized as in [1].

$$
\frac{d y}{d t}=\sigma y^{3}+\gamma y^{2}+\beta y+\alpha
$$

where $\sigma, \gamma, \beta, \alpha \in R$

Left hand side of (6) is represented by $\frac{y_{k+1}-y_{k}}{\varphi(h)}$. For the nonlinear terms $y^{3}, y^{2}$ nonlocal approximation successively are;

$$
y^{3} \approx a y_{k}^{3}+(1-a) y_{k}^{2} y_{k+1} a n d y^{2} \approx b y_{k}^{2}+(1-b) y_{k} y_{k+1} a, b \in R
$$

after plugging in (6), obtained

$$
\frac{y_{k+1}-y_{k}}{\varphi(h)}=a \sigma y_{k}^{3}+(1-a) \sigma y_{k}^{2} y_{k+1}+b \gamma y_{k}^{2}+(1-b) \gamma y_{k} y_{k+1}+\beta y_{k}+\alpha
$$


or

$$
\begin{gathered}
y_{k+1}=\frac{y_{k}+a \varphi(h) \sigma y_{k}^{3}+b \varphi(h) \gamma y_{k}^{2}+\beta \varphi(h) y_{k}+\alpha \varphi(h)}{1-(1-a) \varphi(h) \sigma y_{k}^{2}-(1-b) \varphi(h) \gamma y_{k}} \\
y_{k+1}=F\left(\varphi(h) ; y_{k}\right)
\end{gathered}
$$

with

$$
F(\varphi(h) ; y)=\frac{y+a \varphi(h) \sigma y^{3}+b \varphi(h) \gamma y^{2}+\beta \varphi(h) y+\alpha \varphi(h)}{1-(1-a) \varphi(h) \sigma y^{2}-(1-b) \varphi(h) \gamma y}
$$

To find values of the parameter $a$ and $b$, theorem 1 is applied to (8). Also, by theorems $1-3$, (8) is stable with respect to monotonicity and equilibrium points. According to theorem 1, it should be

$$
\frac{\partial F}{\partial y}(\varphi(h) ; y)>0, F(\varphi(h) ; y)=y \leftrightarrow f(y)=0
$$

\section{Scheme 2.}

For the scheme 2, we consider all terms linear and nonlinear. For the all terms of (6) $y^{3}, y^{2}$, andy nonlocal approximation successively are;

$$
y^{3} \approx a y_{k}^{3}+(1-a) y_{k}^{2} y_{k+1}, y^{2} \approx b y_{k}^{2}+(1-b) y_{k} y_{k+1}, y \approx c y_{k}+(1-c) y_{k+1} a, b, c \in R
$$

after plugging in (6), obtained

$$
\frac{y_{k+1}-y_{k}}{\varphi(h)}=a \sigma y_{k}^{3}+(1-a) \sigma y_{k}^{2} y_{k+1}+b \gamma y_{k}^{2}+(1-b) \gamma y_{k} y_{k+1}+\beta y_{k}+c \beta y_{k}+(1-c) \beta y_{k+1}+\alpha
$$

or

$$
\begin{gathered}
y_{k+1}=\frac{y_{k}+a \varphi(h) \sigma y_{k}^{3}+b \varphi(h) \gamma y_{k}^{2}+\beta \varphi(h) y_{k}+c \varphi(h) \beta y_{k}+\alpha \varphi(h)}{1-(1-a) \varphi(h) \sigma y_{k}^{2}-(1-b) \varphi(h) \gamma y_{k}-(1-c) \varphi(h) \beta} \\
y_{k+1}=F\left(\varphi(h) ; y_{k}\right)
\end{gathered}
$$

with

$$
F(\varphi(h) ; y)=\frac{y+a \varphi(h) \sigma y^{3}+b \varphi(h) \gamma y^{2}+\beta \varphi(h) y+c \varphi(h) \beta y+\alpha \varphi(h)}{1-(1-a) \varphi(h) \sigma y^{2}-(1-b) \varphi(h) \gamma y-(1-c) \varphi(h) \beta}
$$

To find values of the parameter $a$, bandc, theorem 1 is applied to (211). Also, by theorems 1-3, (11) is stable with respect to monotonicity and equilibrium points. According to theorem 1, (9) satisfied.

\section{Scheme 3.}

To compare using time step classical $h$ or $\varphi(h)$, scheme of $\varphi(h)$ denominator function is defined below as in [7-8].

$$
R^{*}:=\max \left\{\left|R_{i}\right|: R_{i}=\left.\frac{d(f(y))}{d y}\right|_{y=\bar{y}(i)} ; i=1: n\right\}
$$


where $\bar{y}^{(i)}$ are fixed points.

$$
\varphi\left(h, R^{*}\right)=\frac{1-e^{R^{*} h}}{R^{*}}=: \varphi(h)
$$

\section{Numerical example}

\section{Hybrid selection equation \& genetics}

$$
\frac{d y}{d t}=k y(1-y)(l-m y)
$$

In (31), $y$ represents the portion of the population, $t$ is the time (measured in generation), $l, m$, and $k$ are constants that depend on genetic characteristic. In our case $l=2, m=1$, and $k=1$ [9].

NSFDM without linear term combination by scheme (1)

$$
\frac{d y}{d t}=y(1-y)(2-y)=y^{3}-3 y^{2}+2 y
$$

After applying (7), we get

$$
\frac{y_{k+1}-y_{k}}{\varphi(h)}=a y_{k}^{3}+(1-a) y_{k}^{2} y_{k+1}-3 b y_{k}^{2}+(1-b)(-3) y_{k} y_{k+1}+2 y_{k}
$$

Equivalently

$$
y_{k+1}=\frac{a \varphi(h) y_{k}^{3}-3 b \varphi(h) y_{k}^{2}+2 \varphi(h) y_{k}+y_{k}}{1-(1-a) \varphi(h) y_{k}^{2}+3 \varphi(h)(1-b) y_{k}}
$$

From (8)

$$
F(\varphi(h) ; y)=\frac{a \varphi(h) y^{3}-3 b \varphi(h) y^{2}+2 \varphi(h) y+y}{1-(1-a) \varphi(h) y_{k}^{2}+3 \varphi(h)(1-b) y_{k}}
$$

From (2.6), we get $a>1$ and $b<0$. If $a$ and $b$ are like these,

$$
F(h ; y)=y \leftrightarrow f(y)=0 \text { for every } h>0 .
$$

NSFDM with linear term combination by scheme (2)

After applying (10) to (14), we get

$$
\frac{y_{k+1}-y_{k}}{\varphi(h)}=a y_{k}^{3}+(1-a) y_{k}^{2} y_{k+1}-3 b y_{k}^{2}+(1-b)(-3) y_{k} y_{k+1}+2 y_{k}+2 c y_{k}+2(1-c) y_{k+1}
$$

Equivalently

$$
y_{k+1}=\frac{a \varphi(h) y_{k}^{3}-3 b \varphi(h) y_{k}^{2}+2 \varphi(h) y_{k}+y_{k}}{1-(1-a) \varphi(h) y_{k}^{2}+\varphi(h)(1-b) y_{k}-2 \varphi(h)(1-c)}
$$

From (11)

$$
F(\varphi(h) ; y)=\frac{a \varphi(h) y^{3}-3 b \varphi(h) y^{2}+2 \varphi(h) y+y}{1-(1-a) \varphi(h) y^{2}+\varphi(h)(1-b) y-2 \varphi(h)(1-c)}
$$

From (9), we get $a>1, b \leq 1, c>1$. If $a, b$ and $c$ are like these,

$$
F(h ; y)=y \leftrightarrow f(y)=0 \text { for every } h>0 .
$$

Denominator function $\varphi(h) ; \bar{y}^{1}=0, \bar{y}^{2}=1, \bar{y}^{3}=2$ are fixed pointes. Thus, by schema (3) $\varphi(h)=\frac{1-e^{-2 h}}{2}$ obtained. 


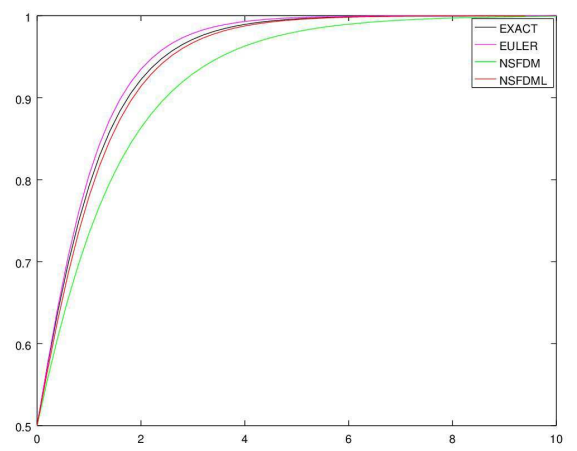

Fig. 1: Numerical approximation of the solution of Eq. (3.2) for $h=0.2$ and $y_{0}=0.5$

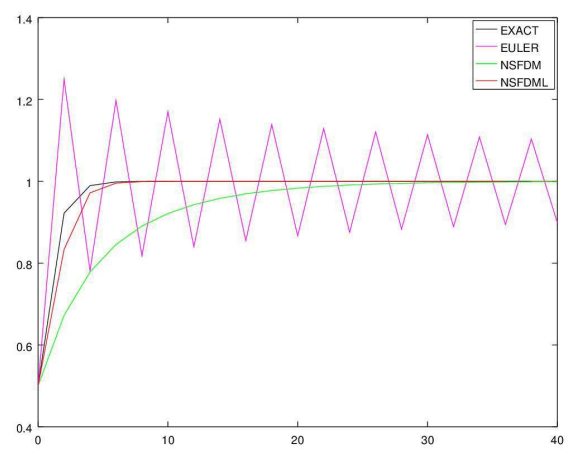

Fig. 3: Numerical approximation of the solution of Eq. (3.2) for $h=2$ and $y_{0}=0.5$.

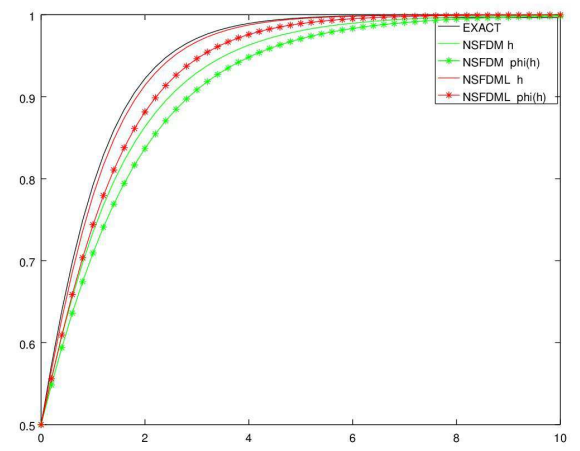

Fig. 5: Numerical approximation of the solution of Eq. (3.2) $\mathrm{h}=0.2$ for $\varphi(h)=\left(1-e^{-2 h}\right) / 2$ and $y_{0}=0.5$.

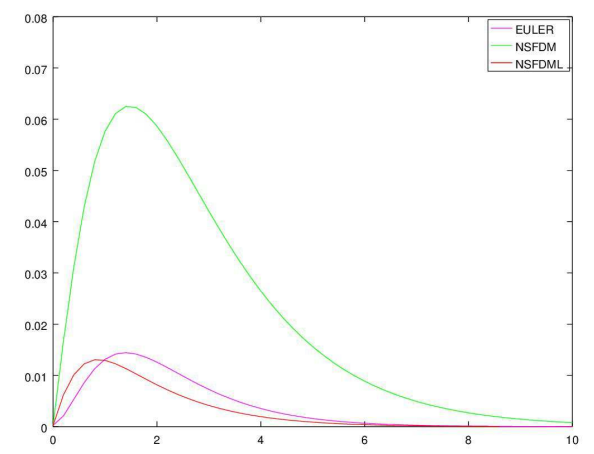

Fig. 2: Numerical errors of the solution of Eq. (3.2) for $h=0.2 a n d y_{0}=0.5$.

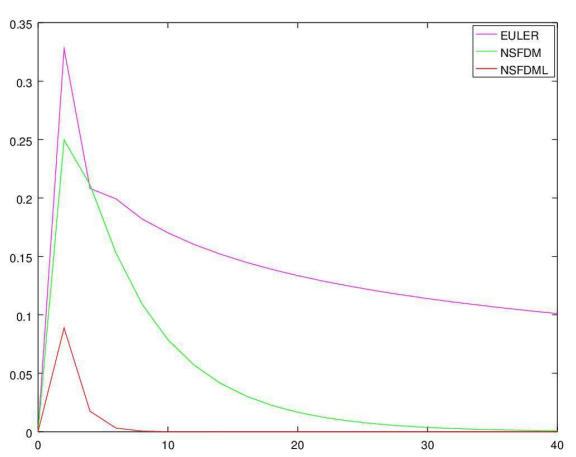

Fig. 4: Numerical errors of the solution of Eq. (3.2) for $h=2 a$ and $y_{0}=0.5$.

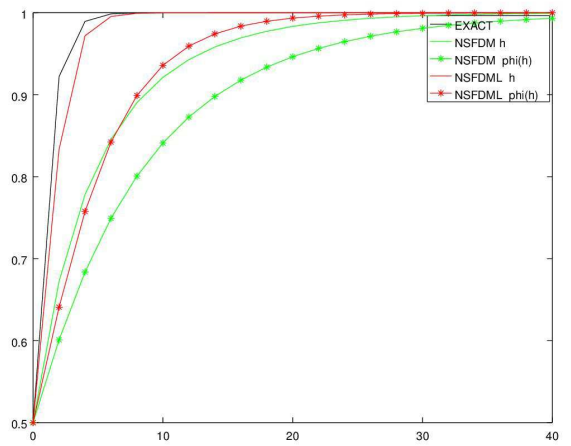

Fig. 6: Numerical approximation of the solution of Eq. (3.2) $\mathrm{h}=2$ for $\varphi(h)=\left(1-e^{-2 h}\right) / 2$ and $y_{0}=0.5$. 
Table 1: Comparison of MATLAB ODE solvers and NSFDMs for eq. (3.2) for $y_{0}=0.5$ based on execution time.

\begin{tabular}{ccc}
\hline \hline Solvers & & Time in second \\
\hline NSFDM & $0.00067(h=0.2)$ & $0.0007(h=2)$ \\
NSFDML & $0.00065(h=0.2)$ & $0.0007(h=2)$ \\
Ode15s & 0.4744 & \\
Ode23s & 0.4022 & \\
\hline
\end{tabular}

\section{Conclusion}

First order stable nonstandard finite difference schema, improved and analyzed in this paper. The improved numerical method is applicable to solving arbitrary first-order differential equation with polynomial right hand side. Making linear term combination in the NSFDM, we get better results in terms of accuracy (see figure 1-4). For denominator function, the graphical result (see, fig. 5-6) shows that using traditional h is the best for the NSFDM. Nonstandard finite difference methods work with big time step (see fig 3-4) but standard difference method fails with big time step. Therefore, NSFDMs have time step freedom. Additionally, MATLAB ODE solvers have same problem which is time step restriction. We experimented with the MATLAB ODE solvers, namely, ode15s and ode 23s. The significant point is that none of these solver is free of step-size restriction. Also, table 1 demonstrates that NSFDMs are faster than MATLAB ODE solvers since NSFDMs have time step freedom but MATLAB ODE solvers do not.

\section{Acknowledgements}

The study is supported by the scientific research project No.2016/01-03 at Bayburt University.

\section{References}

[1] R. Anguelov, J.M.-S. Lubuma, Nonstandard finite difference method by nonlocal approximation, Math. Comput. Simul. 61 (3-6) (2003) 465-475

[2] R. Anguelov, J.M.-S. Lubuma, On the Mathematical Foundation of the Nonstandard Finite Difference Method, in: B. Fiedler, K. Groger, J. Sprekels (Eds.), Proceedings of the International Conference on Differential Equations, EQUADIFF 99, World Scientific, Singapore, 2000, pp. 1401-1403

[3] R. Anguelov, J.M.-S. Lubuma, On the the Nonstandard Finite Difference Method, Keynote address at the Annual Congress of the South African Mathematical Society, Pretoria, South Africa, 16-18 October 2000, Notices S.Afr. Math. Soc. 31 (3) 2000 143-152

[4] R. Anguelov, J.M.-S. Lubuma, Contributions to the Mathematics of the Nonstandard Finite Difference Method and Applications, Num. Methods Partial Differential Equations 17 (5) (2001) 518-543

[5] J.D. Lambert, Numerical Methods for Ordinary Differential Systems, Wiley, Newyork, 1991

[6] R.D. Richtmyer, K.W. Morton, Difference Methods for Initial Value Problems, Interscience, New York, 1967

[7] R.E. Mickens (Ed.), Applications of Nonstandard Finite Difference Schemes, World Scientific, Singapore, 2000.

[8] R.E. Minkens, Nonstandard Finite Difference Schemes for Differential Equations, Journal of Difference Equations and Applications, 2002, 8:9, 823-847

[9] Larson, R. (2006). Calculus: An Applied Approach 9E. Boston: Brooks. 